\title{
La importancia de escuchar a los jóvenes*
}

\section{The importance of listening to the young}

\section{Diana Catalina Castaño Hernández \\ Isabel Cristina Posada Zapata** \\ Érika Tatiana Agudelo Olarte \\ Universidad de Antioquia, Colombia}

Recibido: 17 de agosto de 2017

Revisado: 6 de octubre de 2017 Aceptado: 20 de diciembre de 2017

\section{Resumen}

El objetivo de este estudio fue comprender los modelos de Educación para la Salud en programas de Salud Sexual y Reproductiva (SSR) para jóvenes en Medellín 2004-2010. Se desarrolló a través de metodología cualitativa con teoría fundamentada, realizando entrevistas en profundidad a 8 jóvenes y 12 operadores técnicos (16 entrevistas en la primera fase y 4 en la segunda), las cuales fueron codificadas para extraer las categorías de las cuales emergen los resultados. Se estableció que en los modelos de educación presentes en los programas no se tuvieron en cuenta las necesidades de los jóvenes para su formación en SSR. Existe un amplio desarrollo en políticas públicas de juventud, pero los programas de SSR dirigidos a jóvenes siguen teniendo como único sustento las políticas públicas de salud, por esto se deben tener en cuenta las necesidades de los jóvenes para garantizar más éxito en la ejecución.

Palabras clave: modelos de educación para la salud, salud sexual y reproductiva, jóvenes, operadores, políticas públicas, salud, promoción de la salud.

Artículo de investigación. Citar como: Castaño, H. D., Posada, I. C., \& Agudelo, O. E. (2019). La importancia de escuchar a los jóvenes. Diversitas: Perspectivas en Psicología, 15(1), 27-36. DOI: https://doi.org/10.15332/s1794-9998.2019.0001.02

Correspondencia: Isabel Posada, Facultad Nacional de Salud Pública, Universidad de Antioquia. Correo electrónico: isabel.posada@udea.edu.co 


\section{Abstract}

The objective of this study was to understand the health education models of some sexual and reproductive health programs geared towards young people in Medellín from 2004 to 2010. It was developed using a qualitative methodology with grounded theory, conducting in-depth interviews with 8 young people and 12 technical operators (16 interviews in the first phase and 4 in the second), which were then coded to extract the categories from which the results emerged. It was established based on the results, that in the education models presented in the programs, the needs of young people for their training in SRH were not considered. Althoug, there are a broad development in public youth policies, the SRH programs addressed to young people, continue to have public health policies as their sole and only support. Therefore, the needs of young people must be considered in order to guarantee more success in the execution of this kind of programs.

Keywords: health education models, sexual and reproductive health, young people, operators, public policies, health, health promotion.

\section{Introducción}

Los organismos de salud del mundo han reconocido la importancia de la población joven en el contexto de salud mundial. Se ha centrado la mirada en los jóvenes y se ha encontrado que son una población vulnerable, pero con capacidades y talento, sobre la cual es necesario dirigir acciones concretas de investigación e intervención. La vivencia de una sexualidad insegura y las consecuencias que esta trae al joven ha prendido las alarmas en los organismos de salud y ha ubicado el fenómeno de los embarazos adolescentes en un problema de salud pública, en tanto se reconoce el embarazo juvenil como uno de los factores que mantiene o aumenta el empobrecimiento de las comunidades (Organización Mundial de la Salud [OMS], 2018a).

En Colombia, las cifras en cuanto a embarazo adolescente en los últimos años no han sido positivas. Entre 1990 y 2010 se dio un crecimiento en el porcentaje de mujeres entre $15 \mathrm{y}$ 19 años que han sido madres o están embarazadas, pasando del $12,8 \%$ al $19,5 \%$ entre esos años" (Alcaldía de Medellín, 2012, p. 119). La meta establecida dentro de los Objetivos de Desarrollo del Milenio (ODM) fue que para 2014 y 2015 ese porcentaje estuviera por debajo de 15\%. En Medellín, “el embarazo adolescente ha mostrado una tendencia positiva al pasar en 2008 de 84,2 por mil mujeres, a 71,9 en 2010 para una reducción del 14,6\%; sin embargo, la magnitud del fenómeno sigue siendo alta (Alcaldía de Medellín, 2012, p. 119).

Con relación a este último factor, es importante señalar que algunos de los programas ejecutados en la ciudad de Medellín se han centrado en informar y brindar métodos anticonceptivos a la población juvenil. Así mismo, algunos programas centran su atención en la formación de la autonomía y la ciudadanía juvenil para el desarrollo de proyectos de vida. Bajo este panorama se hace fundamental poder ahondar más en el conocimiento de los programas que se han ejecutado para dicho fin. Esto es, poder indagar cuál ha sido la naturaleza de estas intervenciones y determinar los modelos de Educación para la Salud desde donde se han desarrollado, pues aunque los programas de educación sexual y reproductiva han sido concebidos con el objetivo de procurar mejorar las condiciones de vida de las comunidades y a pesar de los grandes esfuerzos que se han realizado en la promoción de una vida sexual sana y responsable, las cifras de los embarazos adolescentes y los jóvenes infectados con ITS (infecciones de transmisión sexual) señalan que la problemática continúa (OMS, 2018b, 2018c). 
Este trabajo es el resultado de un esfuerzo por retomar los hallazgos y aprendizajes de una investigación sobre modelos de educación en los programas de salud sexual y reproductiva para el período 2004 - 2007 en la ciudad de Medellín realizado en el 2009 y del cual se concluyó que no era factible identificar una propuesta formativa que haya sido diseñada desde un solo modelo de Educación para la Salud. Esto, debido a que los proyectos analizados compartían indicadores de varios modelos, encontrándose incoherencia entre lo formalizado y lo ejecutado. Esto sumado a la tarea que el Estado delegó en la escuela para formar a los jóvenes en materia de educación sexual; la cual, no ha sido asumida adecuadamente por las instituciones educativas, pues a pesar de que existen los proyectos, los docentes no tienen la preparación suficiente y/o la motivación necesaria para llevar a cabo este acompañamiento (Molina, 2009).

Lo anterior ha llevado a que las ideas sobre salud sexual y reproductiva más representativas entre los jóvenes son aquellas que relacionan la sexualidad con tres asuntos: el acto sexual, los métodos anticonceptivos y el embarazo, lo que demuestra que el énfasis que los proyectos han hecho en el abordaje de la salud sexual y reproductiva ha estado centrada en una mirada biomédica, aunque sus marcos conceptuales integren la perspectiva de la sexualidad de una forma más integral. Para la World Health Organization -OMS por sus siglas en español- (2006) la salud sexual y reproductiva es entendida como un estado de bienestar físico, emocional, mental y social relacionado con la sexualidad; no es meramente la ausencia de enfermedad, disfunción o debilidad; la cual requiere un acercamiento positivo y respetuoso hacia la sexualidad y las relaciones sexuales, así como la posibilidad de obtener placer y experiencias sexuales seguras, libres de coerción, discriminación y violencia. Para que la salud sexual se logre y se mantenga, los derechos sexuales de todas las personas deben ser respetados, protegidos y cumplidos.

A pesar de la disminución de la cifras de embarazo adolescente en Medellín entre 2008 y 2010, la problemática continúa y los casos de infecciones de transmisión sexual entre jóvenes son otras de las dificultades con las que se enfrenta Colombia desde hace más de una década. Estas situaciones inciden de manera directa en los índices de deserción escolar, malas condiciones laborales y empobrecimiento sistemático para este grupo poblacional y sus familias, que en muchas ocasiones y a causa de tener que asumir a destiempo roles de adulto para los que posiblemente no están preparados, comienzan a presentar signos de depresión y cambios emocionales que los puede llevar a un detrimento de su salud mental y su bienestar físico (Angulo, Marín, \& Henríquez, 2013).

Es por esto que cobró valor una segunda parte de la investigación para seguir ahondando sobre los hallazgos encontrados inicialmente. Esto a través de la recolección de nueva información, la cual aportó elementos de juicio para la comprensión, teorización, generación de estrategias, datos, información y sugerencias para la formulación, diseño e implementación de programas educativos con un modelo apropiado para la atención y el acompañamiento integral con jóvenes en materia de salud sexual y reproductiva. Sin duda, los hallazgos son una perspectiva más integral para lograr que los programas para jóvenes en materia de sexualidad tengan la repercusión y el resultado esperado.

\section{Metodología}

La presente investigación partió de un diseño cualitativo basada en un enfoque histórico-hermenéutico, desde el cual se concibe al ser humano como un intérprete de la realidad que vive y construye a cada momento. La metodología cualitativa es un modo de encarar el mundo de la interioridad de los sujetos sociales y de las relaciones que establecen con los contextos y otros actores sociales (Galeano, 2004). En esa interioridad se encuentran las realidades subjetivas e intersubjetivas, las cuales se reconocen como objetos legítimos de conocimiento; se comprende desde los actores hasta las lógicas de pensamiento que guían las acciones sociales. Para Galeano (2004) en la investigación social cualitativa hay un especial énfasis en la valoración de lo subjetivo, lo vivencial y la interacción entre los sujetos de la investigación.

El método utilizado fue la teoría fundamentada, aquella que se deriva de datos recopilados de 
manera sistemática y analizados por medio de un proceso de investigación. En este proceso, la recolección de datos, el análisis y la teoría que surge de ellos guardan estrecha relación entre sí y se acercan más a la "realidad" que la teoría derivada de unir una serie de conceptos basados en experiencias o sólo especulando (Corbin \& Strauss, 2002).

Si bien la teoría fundamentada no es un método rígido, si se pueden reconocer tres momentos fundamentales como lo son la codificación, la categorización y la teorización:

- Codificación abierta, donde se codifican todos los elementos surgidos de las primeras entrevistas, dichos códigos se agrupan en categorías emergentes denominadas Descriptivas; este primer momento de análisis es descriptivo.

- En un segundo momento denominado analítico, se realiza la codificación axial en donde se relacionan esas primeras categorías con unas subcategorías, en dicha codificación se van enlazando y relacionando las categorías de acuerdo con sus propiedades y dimensiones, y de la cual surgen las categorías analíticas.

- Finalmente se realiza una codificación selectiva, emergen las categorías interpretativas, se integran y refinan las categorías, para así llegar a una teoría (teorización). Este tercer momento es interpretativo (Corbin \& Strauss, 2002).

Se utilizó el muestreo teórico, el cual no estaba predeterminado antes de iniciar la investigación, sino que fue evolucionando y construyéndose a partir del análisis. Así, se estableció la metodología que permitiera indicar las lagunas o vacíos existentes; el muestreo y el análisis ocurrieron de manera secuencial y este último fue el que guio la recolección de los datos (Corbin \& Strauss, 2002).

La técnica de recolección utilizada fue la entrevista en profundidad, permitiendo al investigador solicitar, mediante una serie de preguntas, información sobre una situación determinada. Se crearon dos guiones de entrevista, uno para los operadores técnicos de programas de salud sexual y reproductiva; y el segundo guion para beneficiarios jóvenes de programas de salud sexual y reproductiva. Las preguntas giraron en torno a temas como: la información previa para la formulación de los programas, la manera en que se había llegado al programa, los objetivos, las etapas de los programas, la metodología empleada, el personal profesional que acompañó el proceso, los resultados del programa.

La muestra se fue conformando en el proceso investigativo a partir de las conceptualizaciones encontradas. Esto, con el fin de contener suficiente información que permitiera llegar a la saturación teórica, punto que indicó que se debía suspender la recolección. La muestra total de la primera parte fue de 16 participantes: 10 operadores y 6 jóvenes. En la segunda parte se contó con 4 participantes: 2 operadores y 2 jóvenes. En total, se entrevistaron 20 personas ( 12 operadores y 8 jóvenes) a los cuales se les facilitó el consentimiento informado para su firma actuando bajo los lineamientos de la resolución 8430 de 1993 del Ministerio de Salud de Colombia sobre la investigación con seres humanos, garantizando la integridad física y mental de los participantes (Ministerio de Salud, 1993). Para la investigación el riesgo se estimó como mínimo, ya que, si bien en ningún momento se tenía previsto hacer intervención, sí se tuvieron en cuenta los posibles riesgos psicosociales que se derivarían del discurso voluntario de los participantes.

Las entrevistas se transcribieron y luego fueron codificadas y analizadas. Después de revisar las categorías descriptivas de las últimas cuatro entrevistas, a la luz de las categorías descriptivas de las 16 entrevistas anteriores, se logró una saturación de la información que permitió dilucidar dos categorías analíticas y que fueron el producto de una relación de contenidos y temas que habían emergido a lo largo de las dos investigaciones y que comenzaban a descubrir actores, causas y consecuencias del fenómeno investigado, mostrando un hilo conductor de los hallazgos y las ideas más relevantes.

\section{Resultados y discusión}

Tras el desarrollo del proceso de categorización emergieron dos categorías interpretativas las cuales fueron el resultado de un esfuerzo teórico y de análisis de la información otorgada por los entre- 
vistados y su relación con la realidad estudiada, que para este caso fueron los modelos educativos de salud sexual y reproductiva. Sin embargo, en este artículo se enfatizará en la categoría denominada La importancia de escuchar a los jóvenes, ya que fue la que permitió entender el contexto social del desarrollo de esos modelos, a partir de experiencias y conocimientos previos de los jóvenes, permitiendo develar la manera como ellos perciben los modelos actuales y entregando pistas sobre la posible construcción de modelos futuros. De esta categoría se desarrollan tres subcategorías: los jóvenes sujetos de derechos; el arte: la estética juvenil por excelencia; y la necesidad de una educación creativa.

\section{Los jóvenes sujetos de derechos}

El común denominador de las respuestas otorgadas durante las entrevistas da cuenta de una población que ha ido entendiendo sus necesidades y adquiriendo una capacidad de juicio y criterio que los empodera, y les facilita la reclamación de sus propios derechos.

No deja de ser paradójica la preeminencia del modelo de educación informativo para la salud sexual y reproductiva, cuando existe un conglomerado jurídico en materia juvenil, con una alta valoración por los derechos políticos, económicos y culturales, que comenzó a implementarse, entre otras, como consecuencia de una serie de circunstancias históricas que conmocionaron al país, especialmente a la Ciudad de Medellín durante la década de los años 80 y 90 por el fenómeno del narcotráfico.

El fenómeno del narcotráfico originó caos y desorden, donde los protagonistas fueron los jóvenes, que bajo condiciones económicas precarias y el auge de la cultura de la ilegalidad, auspiciada por sus propias familias, se fueron convirtiendo en sicarios a sueldo de los barrios más pobres, cerrando las posibilidades y las oportunidades para el resto de los jóvenes que querían hacer algo diferente.

Garcés (2008) en su estudio “Juventud, investigación y saberes. Estado del arte de las investigaciones sobre la realidad juvenil en Medellín. 2004 - 2006" muestra que las lógicas instrumentales de las relaciones afectivas, la posibilidad de comprarlo todo, incluso el afecto y el amor, entre otras manifestaciones, llevaron a que al cabo de los años y cuando el deterioro cultural y social fue notorio, un grupo de personas se pusieron en la tarea de mostrar otras realidades juveniles y fue por eso, que la ciudad de Medellín se convirtió en líder protagonista de la movilización del desarrollo y la implementación de la Política Pública de Juventud (Garcés, 2008) 1 .

Se destaca entonces que muchos jóvenes han ido introyectando el discurso de los derechos y de entenderse como actores de ciudadanía y por eso de manera reiterada, insisten en el bienestar que les genera una educación en salud sexual y reproductiva fundamentada en principios de igualdad y respeto, donde los identifiquen como productores de cultura, no sólo como consumidores de ella y sujetos de saberes y verdades impartidas por los adultos.

...y también el objetivo y la capacidad que adquirimos con ese proyecto era de ser jóvenes de reproducción de la información y esparcirlo en todo el colegio (E2B-15C16)... y la relación que manteníamos entre docentes y estudiantes no era tanto como docente estudiante sino como un poco más cercana (E2B-66C67)... pero la estrategia que más ha utilizado es plantearnos dilemas en la que pues los jóvenes, como sujetos ejemplares, deben tomar alguna decisión (E2B-132C133)

Además de esto, los participantes manifestaron que en los programas se propuso darle voz a los jóvenes en la construcción de significados y para esto fue determinante que los docentes no se pusieran en una posición de poder por el supuesto saber:

...yo pienso que era bacano porque estábamos hablando desde la horizontalidad, desde la metodología de pares ¡claro! (E3B-58C59)

...siempre hablaban los jóvenes, si por ejemplo yo me hacía una interrogación la respondía otro joven el cual tenía como el saber o tenía esa respuesta. (E3B-42C43)

$1 \quad$ Ley 375 de 1997, Acuerdo 02 de 2000 y Acuerdo 074 de 2007. 
...excelente porque entonces se veía el empoderamiento de los jóvenes o sea se veía como la base que tenían en el tema de sexualidad (E3B-52C53)

Si a los jóvenes se les da autonomía se logra su empoderamiento en el tema sexual, porque en términos generales, es una población que desea figurar y ser protagonista sin dejar de reconocer y valorar el acompañamiento y la orientación de los adultos; siempre y cuando se les reconozca y respete su lugar de saber, en igualdad de condiciones, siendo importante, además, encontrar un vínculo afectivo con el adulto que permitiera establecer lazos de confianza y seguridad en el joven.

...era muy charro porque entonces nosotros decíamos que no teníamos tallerista, era muy charro y decíamos: tráiganos a nosotros el tema, que nosotros no necesitamos tallerista (E3B-50C51)

...la profesora de ética que es con la que más nos relacionamos (E2B-123C124) Hay confianza con esa docente (E2B-124C125)

...esa profesora pues para mí es excelente ya que maneja la relación entre estudiantes muy bien (E2B-128C129)

...y ella como que nos orienta más fácilmente de una forma más comprensiva (E2B-130C131).

En medio de este panorama, llamó la atención que las Políticas Públicas de Salud, aún no hayan retomado las Políticas Públicas de Juventud, que como se dijo anteriormente, han logrado recoger el sentir y la voz juvenil de los últimos tiempos.

\section{El arte: la estética juvenil por excelencia}

Al entrevistar a los jóvenes sobre los programas de salud sexual y reproductiva, la gran mayoría coincidió en resaltar de manera positiva el trabajo donde se privilegiaba el desarrollo artístico a partir de las propias capacidades y potencialidades. En este sentido Molina (2009) reiteraba que "desde la postura de los operadores técnicos, la mejor metodología para trabajar este tipo de procesos con jóvenes es aquella donde se privilegia la elaboración personal del sujeto por encima de otro tipo de intervenciones" (p. 137).

“...Era algo muy chévere (E3B-44C45) y al final del proyecto hicimos un video que era como montando una obra de teatro (E2B-37C38) (...) y en la obra de teatro hablábamos sobre la diversidad de géneros y orientaciones sexuales (E2B-39C40)"

Quizás el significado que para los jóvenes representa el arte, es lo que pueda explicar la demanda que hicieron frente a la intensidad horaria del componente artístico dentro de algunos de los proyectos de salud sexual y reproductiva que contemplaron dicho componente. Esa necesidad también fue percibida por parte de las personas que diseñaron y operaron el proyecto de Salud Sexual y Reproductiva con el énfasis de los núcleos creativos:

"un ejemplo si un grupo de jóvenes quiere constituir un grupo de teatro que se les dé la posibilidad de más horas de capacitación en esa línea técnica" (E10-122C123).

Las entrevistas develaron dos tipos de enfoques a la hora de desarrollar los programas de salud sexual y reproductiva para jóvenes: una de tipo ecológico, económico y político, y otro de tipo informativo y preventivo como bien los describe Serrano (1990). Tanto el uno como el otro, reconocen la importancia del arte, pero la diferencia entre ambos radica principalmente en el abordaje y el sentido que le dieron a este. Para el primer enfoque, el arte se convirtió en parte de la elaboración personal del sujeto joven y por eso es una producción que surgió de ellos mismos, como una iniciativa, un consenso y un deseo:

“...Eh pues me parece que para todo había su tiempo cuando había que tomar las cosas las tomábamos pero ya la obra de teatro ya fue como dijo mi compañero, fue una elección de nosotros" (E2B-109C110).

Para el segundo enfoque preventivo e informativo, la creación artística se convirtió en el instrumento, en el medio para lograr un fin que generalmente 
está dado por un deseo de adultos, que tienen un mensaje para entregar sobre la prevención del embarazo adolescente, la planificación, o el tema de interés, y precisamente así lo deja ver uno de los operadores del proyecto en sus afirmaciones:

“...es decir, las herramientas que yo manejo son apoyadas en el juego y en el teatro para llevar lo que es la educación a nivel de infecciones de transmisión sexual, embarazo en adolescentes, embarazo no deseado y violencia sexual" (E4O-12C13).

La distinción entre el uso del arte en ambos enfoques es clara, pero vale la pena retomar el criterio del arte creador como fin en sí mismo, como elemento central que ayuda a encontrar respuestas a los interrogantes que los jóvenes, se formulan día a día frente a los problemas y sentimientos que afloran en ellos. Que mejor manera de encontrar caminos y salidas a las preguntas para estos jóvenes, que la posibilidad de encontrarse, mirarse y verse en los otros, darse cuenta de que sus angustias y problemas no son únicos y que existe la manera de construir y hacer proyectos compartiendo sueños.

[...] la mayoría de clubes juveniles trabajan desde el arte (E3B-18C19) o sea se trataba de buscar diferentes artes, como éramos subgrupos, entonces habían diferentes artes habían grafiteros, teatreros (E3B-20C21) solo fue el arte y cada 8 días cambiábamos de actividad artística (E3B-27C28) y era como el proceso de arte y sexualidad, yo pienso que eso se debería de enfocar, eso se debería de translucir (E3B-81C82)

Es entonces que en la vía de retomar la creación artística, el juego y la lúdica como parte de los lineamientos que guíen los programas de Salud Sexual y Reproductiva para jóvenes de la ciudad, se ha visto en la presente investigación, que existe una ruta trazada en el actual Plan Estratégico de Desarrollo Juvenil 2007 (Concejo de Medellín, 2006), en el cual se presenta un Escenario 2: Sobre Identidades, Experiencias y Expresiones juveniles para el reconocimiento de prácticas y representaciones juveniles, que dictamina: "promover en los y las jóvenes la apropiación crítica del cuerpo, el tiempo, el territorio y los bienes culturales, para la construcción ética de sus proyectos de vida...".

Parece ser que la Política Pública plasmada en el Plan Estratégico de Desarrollo Juvenil desde el año 2007 (Concejo de Medellín, 2006) llegó a su fin (tuvo vigencia hasta el año 2015), sin el eco necesario para ayudar a trazar un modelo educativo en Salud Sexual y Reproductivo más acorde con las necesidades y demandas de los jóvenes.

Actualmente, la demanda de los jóvenes en materia de Salud Sexual y Reproductiva parece estar apuntando a una necesidad más profunda de relacionamiento con el otro, que pasa básicamente por los afectos, los sentimientos y las emociones. El hecho de que el arte esté de por medio, sumado a algunos testimonios de los mismos jóvenes y operadores de programas, parece estar indicando que la búsqueda de los jóvenes trasciende el deseo de adquirir preservativos para evitar enfermedades y embarazos tempranos, se están preguntando por los asuntos de roles de género, las relaciones familiares, con sus pares y amigos, entre otros, y adquieren el concepto de valoración y respeto por el propio cuerpo y el de los demás.

...pues yo pienso por mi parte me sirvió mucho porque ya eso como que afianza la relación entre una familia (E2B-93C94) porque hay muchas familias que tienen cierto tapujos al hablar de eso (E2B-92C93) pero ya con este proyecto comprendí que sexualidad toca mucho ese aspecto de la vida especialmente lo social (E2B-112C113)

...y entonces la sexualidad tienen que ver mucho ahí ya a la hora de relacionarnos con los demás, hay que valorar nuestro cuerpo y valorar el de los demás (E2B-118C119).

Es por esto, por lo que los pocos programas que se han preguntado por entender e investigar realmente sobre la sexualidad y su papel en el desarrollo del ser joven, han adoptado otros estilos y otras estrategias relacionadas con el juego y el arte, no como instrumento metodológico, sino como fin en sí mismo. 


\section{La necesidad de una educación creativa}

Como se vio a lo largo de la información analizada, parece que las reflexiones que se tienen para el modelo educativo que se imparte hoy en la mayoría de las aulas de clase, vale también para los modelos de educación en materia de Salud Sexual y Reproductiva para jóvenes:

“...desde la parte directiva de las instituciones en general, (E10-115C116) a veces hay una posición de mucho autoritarismo, de mucha unilateralidad que impide muchas veces... eh primero que el trabajo se haga de acuerdo al contexto juvenil [...]" (E10-116C117).

Frente a lo anterior, se indica que la escuela y los modelos de educación, deberían estar buscando estrategias y contenidos para lograr el fin de ayudar en la formación de sujetos autónomos. Pero para eso, es imprescindible que los adultos crean en la capacidad de ellos como sujetos creadores de cultura y de su propia historia. Las entrevistas realizadas a jóvenes reivindicaron una educación que facilite el desarrollo de habilidades para la vida, la comunicación y la comprensión con otros pares, que suscite preguntas sobre el cuerpo, el afecto y la sexualidad que los involucre como individuos éticos, y que pueda ser incluso a partir de la estética y el arte; como diría Serrano (1990), un modelo de educación amplio e integral que comprenda lo ecológico, político y económico en su concepción:

“...Me pareció una experiencia excelente que nos puede ayudar a muchos en la formación de vidas (E2B-74C75) entonces la gente empieza a hacer un proyecto de vida así sea muy metafórico pero empieza a hacer proyecto de vida [...]" (E3B-96C97)

Sobre los programas de salud sexual y reproductiva, se expresaron críticas por parte de los entrevistados, y se debían en parte a los enfoques de corte preventivo. Esto debido a que, según los operadores de programas, las competencias deberían estar relacionadas con el saber teórico:

“...entonces la atención en salud sigue centrándose mucho en el adolescente, en los métodos de planificación para que no se embarace o en prevenir las infecciones de transmisión sexual” (E10-85C86).

En este sentido Molina (2009) expresa:

La tarea que el Estado delegó en la escuela para formar a los jóvenes en materia de educación sexual no ha sido asumida adecuadamente por las instituciones educativas, pues a pesar de que existen los proyectos, el docente no tiene la preparación suficiente y/o la motivación necesaria para llevar a cabo este acompañamiento (p. 138).

Es por esto, por lo que se requiere de un modelo que le apunte a la generación de lugares para la imaginación y la creación como desarrollo del ser, sin el temor a la censura y el señalamiento:

“...en la metodología de los talleres uno debe siempre trabajar desde ahí no desde un enfoque tradicional: de vamos a hablar de conceptos y hablemos desde lo teórico no, yo creo que eso está revaluado y más con los chicos" (E10-141C142).

Todos los días la población joven le está diciendo a los otros jóvenes, a los adultos, a la sociedad y al Estado lo que quiere, lo que desea, lo que le importa, lo que los hace vibrar, lo que los hace llorar y sentirse tristes.

Sin embargo, está presente el reto de conocer a la juventud actual. Croce (2012), en su texto "Nuevas realidades juveniles en América Latina”, afirma que:

Las y los jóvenes viven híper- estimulados por imágenes, sonidos, movimientos, luces, vibraciones... muy intensas, fugaces, impactantes. En éste contexto se les hace difícil mantener mucho tiempo la atención, sobre todo cuándo se trata de situaciones en las que se combinan esfuerzo con constancia. La educación formal es uno de los espacios que más está sufriendo el impacto de estos cambios culturales profundos (p. 3).

Savater (2012) en su texto "Ética de la Urgencia" añade: 
Hoy en día, con la televisión y con Internet, la prioridad ya no puede ser informar, si no orientar al alumno a través del laberinto de información que le está bombardeando constantemente. Así que la educación ya no puede centrarse en informar [...] (p. 32).

Finalmente se resalta que, interpretar el sentir, procurar entender en qué consisten las necesidades reales de los jóvenes de hoy, es requisito indispensable para construir ese modelo educativo para la educación en salud sexual y reproductiva desde un enfoque político, económico y ecológico como el que propone Serrano (1990). Lo demás serán esfuerzos para reproducir programas en cumplimiento de un deber legal, que por no tener en cuenta a la población para la cual van dirigidos, terminará perpetuando el desencanto y el desinterés de los jóvenes por atender un discurso de adultos interesados en disminuir el embarazo adolescente y las enfermedades de transmisión sexual, pero que a ellos poco o nada les dice de sus verdaderas búsquedas y estilos.

\section{Conclusiones}

A pesar del amplio desarrollo en políticas públicas de juventud para la ciudad de Medellín, con un claro modelo educativo que propende por lo político, económico, ecológico tal como lo presenta Serrano, la concepción de los programas de salud sexual y reproductiva que se dirigen a la población joven, siguen teniendo como único sustento, las políticas públicas de salud, desconociendo las necesidades del grupo poblacional específico para el cual van enfocados.

La mayoría de los jóvenes manifiestan desinterés y señalan deficiencias en los programas de Salud sexual y Reproductiva única y exclusivamente de corte informativo y preventivo como el caso de los servicios amigables.

Los jóvenes muestran un interés especial por el desarrollo afectivo y en ese sentido demandan espacios para el encuentro, el intercambio de experiencias con pares, la conversación y la escucha real de los adultos que acompañan sus procesos formativos.
El arte entendido como fin en sí mismo, y no como instrumento o medio para alcanzar otro propósito, es una propuesta formativa que le permite a los jóvenes la apropiación crítica del cuerpo, el tiempo, el territorio y los bienes culturales, para la construcción ética de sus proyectos de vida.

La salud sexual como componente central que hace parte de la emocionalidad y la experiencia vital de la población joven no parece constituir un interés general para la comunidad educativa, institucional, local e internacional.

\section{Agradecimientos}

A la estrategia de sostenibilidad CODI de la Universidad de Antioquia por su apoyo para la realización del artículo

\section{Referencias}

Alcaldía de Medellín. (2012). Línea de base del índice de Desarrollo Juvenil, Medellín, 2011-2012. Medellín: Alcaldía de Medellín. Recuperado de https://issuu.com/medellinjoven/docs/ indice_de_desarrollo_juvenil

Angulo, C. V., Marín, E. V., \& Henríquez, G. E. (2013). Embarazo adolescente, un cambio de hábitos y comportamientos? Revista CES Salud Pública, 4, 84-91.

Concejo de Medellín. (2006). Acuerdo Municipal 76 de 2006, Por el cual se adopta el Plan Estratégico Municipal de Desarrollo Juvenil de Medellín 2007-2015. Medellín: El Concejo.

Corbin, J. \& Strauss, A. (2002). Bases de la investigación cualitativa. Técnicas y procedimientos para desarrollar la teoría fundamentada. Medellín: Editorial Universidad de Antioquia.

Croce, A. (2012). Nuevas realidades juveniles en América Latina. Revista América Latina en Movimiento, 477. Recuperado de https://www. alainet.org/es/active/56602 
Galeano, M. E. (2004). Estrategias para investigación social cualitativa: el giro de la mirada. Medellín: La Carreta Editores.

Garcés, A. P. (2008). Juventud, investigación y saberes. Estado del arte de las investigaciones sobre la realidad juvenil en Medellín. 2004-2006. Medellín: Sello Editorial Universidad de Medellín.

Ministerio de Salud. (1993). Resolución 8430 de 1993: por la cual se establecen las normas científicas, técnicas y administrativas para la investigación en salud. Bogotá: Ministerio de Salud.

Molina, D. P. (2009). Perspectiva de algunos actores sobre los modelos de educación para la salud subyacentes en programas de salud sexual y reproductiva dirigidos a adolescentes, Medellín 2004-2007. [Tesis de maestría]. Medellín: Universidad de Antioquia.

Organización Mundial de la Salud, OMS (2018a). Salud Mental del Adolescente. Disponible en: https://www.who.int/es/news-room/ fact-sheets/detail/adolescent-mental-health Consultado: 8 de diciembre de 2018.
Organización Mundial de la Salud (2018b). El embarazo en la adolescencia. Disponible en: https:// www.who.int/es/news-room/fact-sheets/detail/adolescent-pregnancy Consultado: 8 de diciembre de 2018.

Organización Mundial de la Salud (2018c). Infecciones de transmisión sexual. Disponible en: https://www.who.int/es/news-room/factsheets/detail/sexually-transmitted-infections(stis). Consultado: 8 de diciembre de 2018.

Savater, F. (2012). Ética de la urgencia. México: Ariel Lugar de Edición.

Serrano, M. I. (1990). Educación para la salud y participación comunitaria. Madrid: Ediciones Díaz de Santos.

World Health Organization. (2006). Defining sexual health: report of a technical consultation on sexual health. Recuperado de http://www.who. int/reproductivehealth/topics/gender_rights/ defining_sexual_health.pdf 